\title{
Study of charged hadron multiplicities in charged-current neutrino-lead interactions in the OPERA detector
}

N. Agafonova ${ }^{1}$, A. Aleksandrov ${ }^{2}$, A. Anokhina
, S. Aoki N. D’Ambrosio ${ }^{13}$, G. De Lellis ${ }^{2,11}$, M. De Serio ${ }^{14,15}$, P. del Amo Sanchez ${ }^{16}$, A. Di Crescenzo ${ }^{2,11}$, D. Di Ferdinando ${ }^{17}$, N. Di Marco ${ }^{13}$, S. Dmitrievski ${ }^{8}$, M. Dracos ${ }^{18}$, D. Duchesneau ${ }^{16}$, S. Dusini ${ }^{7}$, T. Dzhatdoev ${ }^{3}$, J. Ebert ${ }^{19}$, A. Ereditato ${ }^{5}$, R. A. Fini ${ }^{15}$, F. Fornari ${ }^{17,20}$, T. Fukuda ${ }^{21}$, G. Galati ${ }^{2,11}$, A. Garfagnini ${ }^{7,10}$, V. Gentile ${ }^{36}$, J. Goldberg ${ }^{22}$, Y. Gornushkin ${ }^{8}$, S. Gorbunov ${ }^{12}$, G. Grella ${ }^{9}$, A. M. Guler ${ }^{23, a}$, C. Gustavino ${ }^{25}$, C. Hagner ${ }^{19}$, T. Hara ${ }^{4}$,

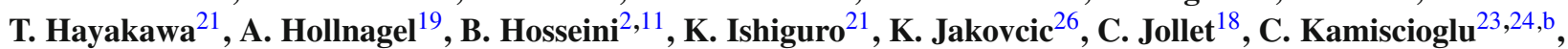
M. Kamiscioglu ${ }^{23}$, S. H. Kim ${ }^{27}$, N. Kitagawa ${ }^{21}$, B. Klicek ${ }^{28}$, K. Kodama ${ }^{29}$, M. Komatsu ${ }^{21}$, U. Kose ${ }^{7}$, I. Kreslo ${ }^{5}$,

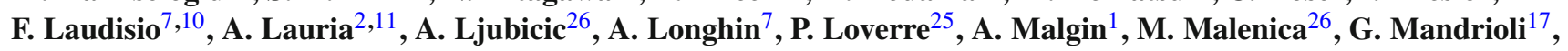
T. Matsuo $^{30}$, V. Matveev ${ }^{1}$, N. Mauri ${ }^{17,20}$, E. Medinaceli ${ }^{7,10}$, A. Meregaglia $^{18}$, S. Mikado ${ }^{31}$, M. Miyanishi ${ }^{21}$, F. Mizutani ${ }^{4}$, P. Monacellii ${ }^{25}$, M. C. Montesi ${ }^{2,11}$, K. Morishima ${ }^{21}$, M. T. Muciaccia ${ }^{14,15}$, N. Naganawa ${ }^{21}$, T. Naka ${ }^{21}$, M. Nakamura ${ }^{21}$, T. Nakano ${ }^{21}$, K. Niwa ${ }^{21}$, N. Okateva ${ }^{12}$, S. Ogawa ${ }^{30}$, K. Ozaki ${ }^{4}$, A. Paoloni ${ }^{32}$, L. Paparella ${ }^{14,15}$, B. D. Park ${ }^{27}$, L. Pasqualini ${ }^{17,20}$, A. Pastore ${ }^{14,15}$, L. Patrizii ${ }^{17}$, H. Pessard ${ }^{16}$, D. Podgrudkov ${ }^{3}$, N. Polukhina ${ }^{12,33}$, M. Pozzato ${ }^{17,20}$, F. Pupilli ${ }^{7}$, M. Roda ${ }^{7,10}$, T. Roganova ${ }^{3}$, H. Rokujo ${ }^{21}$, G. Rosa ${ }^{25}$, O. Ryazhskaya ${ }^{1}$, O. Sato ${ }^{21}$, A. Schembri ${ }^{13}$, I. Shakirianova ${ }^{1}$, T. Shchedrina ${ }^{12}$, H. Shibuya ${ }^{30}$, E. Shibayama ${ }^{4}$, T. Shiraishi ${ }^{21}$, S. Simone ${ }^{14,15}$, C. Sirignano ${ }^{7,10}$, G. Sirri ${ }^{17}$, A. Sotnikov ${ }^{8}$, M. Spinetti ${ }^{32}$, L. Stanco ${ }^{7}$, N. Starkov ${ }^{12}$, S. M. Stellacci ${ }^{9}$, M. Stipcevic ${ }^{28}$,

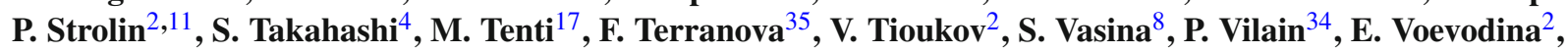

L. Votano ${ }^{32}$, J. L. Vuilleumier ${ }^{5}$, G. Wilquet ${ }^{34}$, B. Wonsak $^{19}$, C. S. Yoon ${ }^{27}$

${ }^{1}$ INR - Institute for Nuclear Research of the Russian Academy of Sciences, 117312 Moscow, Russia

2 INFN Sezione di Napoli, 80125 Naples, Italy

${ }^{3}$ SINP MSU - Skobeltsyn Institute of Nuclear Physics, Lomonosov Moscow State University, 119991 Moscow, Russia

${ }^{4}$ Kobe University, 657-8501 Kobe, Japan

${ }^{5}$ Laboratory for High Energy Physics (LHEP), Albert Einstein Center for Fundamental Physics, University of Bern, 3012 Bern, Switzerland

${ }^{6}$ Faculty of Arts and Science, Kyushu University, Fukuoka, Japan

${ }^{7}$ INFN Sezione di Padova, 35131 Padua, Italy

8 JINR - Joint Institute for Nuclear Research, 141980 Dubna, Russia

9 Dipartimento di Fisica dell'Università di Salerno and "Gruppo Collegato" INFN, 84084 Fisciano, Salerno, Italy

${ }^{10}$ Dipartimento di Fisica e Astronomia dell’Università di Padova, 35131 Padua, Italy

${ }^{11}$ Dipartimento di Fisica dell'Università Federico II di Napoli, 80125 Naples, Italy

12 LPI - Lebedev Physical Institute of the Russian Academy of Sciences, 119991 Moscow, Russia

13 INFN-Laboratori Nazionali del Gran Sasso, 67010 Assergi, L'Aquila, Italy

${ }^{14}$ Dipartimento di Fisica dell'Università di Bari, 70126 Bari, Italy

15 INFN Sezione di Bari, 70126 Bari, Italy

${ }^{16}$ LAPP, Université Savoie Mont Blanc, CNRS/IN2P3, 74941 Annecy-le-Vieux, France

17 INFN Sezione di Bologna, 40127 Bologna, Italy

18 IPHC, Université de Strasbourg, CNRS/IN2P3, 67037 Strasbourg, France

${ }^{19}$ Hamburg University, 22761 Hamburg, Germany

${ }^{20}$ Dipartimento di Fisica e Astronomia dell'Università di Bologna, 40127 Bologna, Italy

${ }^{21}$ Nagoya University, 464-8602 Nagoya, Japan

22 Department of Physics, Technion, 32000 Haifa, Israel

${ }^{23}$ METU - Middle East Technical University, 06800 Ankara, Turkey

24 Ankara University, 06560 Ankara, Turkey

25 INFN Sezione di Roma, 00185 Rome, Italy

${ }^{26}$ Rudjer Boskovic Institute, 10002 Zagreb, Croatia

27 Gyeongsang National University, 900 Gazwa-dong, Jinju 660-701, South Korea

${ }^{28}$ Center of Excellence for Advanced Materials and Sensing Devices, Ruder Bošković Institute, 10002 Zagreb, Croatia

29 Aichi University of Education, 448-8542, Kariya Aichi, Japan

${ }^{30}$ Toho University, 274-8510 Funabashi, Japan

31 Nihon University, 275-8576, Narashino Chiba, Japan

32 INFN - Laboratori Nazionali di Frascati dell'INFN, 00044 Frascati, Rome, Italy 
${ }^{33}$ Moscow Engineering Physical Institute Moscow, Moscow, Russia

${ }^{34}$ IIHE, Université Libre de Bruxelles, 1050 Brussels, Belgium

35 Dipartimento di Fisica dell'Università di Milano-Bicocca, 20126 Milan, Italy

${ }^{36}$ Gran Sasso Science Institute, L'Aquila, Italy

Received: 23 June 2017 / Accepted: 27 December 2017 / Published online: 24 January 2018

(C) The Author(s) 2018. This article is an open access publication

\begin{abstract}
The OPERA experiment was designed to search for $v_{\mu} \rightarrow v_{\tau}$ oscillations in appearance mode through the direct observation of tau neutrinos in the CNGS neutrino beam. In this paper, we report a study of the multiplicity of charged particles produced in charged-current neutrino interactions in lead. We present charged hadron average multiplicities, their dispersion and investigate the KNO scaling in different kinematical regions. The results are presented in detail in the form of tables that can be used in the validation of Monte Carlo generators of neutrino-lead interactions.
\end{abstract}

\section{Introduction}

The multiplicity distribution of charged hadrons is an important characteristic of the hadronic final states in hard scattering processes. Since it reflects the dynamics of the interaction, it has been extensively studied in cosmic rays, fixed target and collider experiments [1-7]. These data are useful to improve models of particle production which are used in Monte Carlo (MC) event generators.

In this paper, we report the result on charged hadron production initiated in charged-current $v_{\mu}$ interactions in the OPERA target. The basic unit that constitutes the target is the emulsion cloud chamber (ECC) detector which is a stack of nuclear emulsion films acting as high precision trackers interleaved with lead plates that provide a massive target. The excellent spatial resolution of nuclear emulsion allows the determination of the event topology and the measurement of charged particle trajectories. Therefore, it is well suited for the investigation of the moments of the charged particles multiplicity distribution. However, only few studies of charged particle multiplicity in neutrino-nucleon interactions were made using the nuclear emulsion technology [7-10].

In the following, a short description of the experimental setup and of the procedure used to locate neutrino interactions in the target is given; the data sample and the analy-

B. Hosseini: Now at Sezione INFN di Cagliari

U. Kose: Now at CERN

E. Medinaceli: Now at Osservatorio Astronomico di Padova

M. Roda: Now at University of Liverpool.

a e-mail: ali.murat.guler@cern.ch

b e-mail: Cagin.Gunes@ ankara.edu.tr sis procedure are presented. Then, multiplicity moments and investigation of KNO scaling [11] in different kinematical regions are presented in a form suitable for the validation of $\mathrm{MC}$ generators of neutrino-lead interactions.

\section{Experimental procedure}

The OPERA experiment has been designed for the observation of $v_{\mu} \rightarrow v_{\tau}$ oscillations in appearance mode in the CNGS (CERN Neutrinos to Gran Sasso) neutrino beam [1214]. The detector was located at the underground Gran Sasso Laboratory (LNGS) in Italy. It was exposed to the CNGS muon neutrino beam with mean energy $17 \mathrm{GeV}$ at a distance of $730 \mathrm{~km}$. OPERA reported the discovery of $v_{\tau}$ appearance with a significance of 5.1 $\sigma$ [15]. The OPERA detector is a hybrid setup consisting of electronic detectors and a massive lead-emulsion target segmented into ECC units, called bricks. The detector is composed of two identical Super-Modules, each of which has a target section followed by a muon spectrometer which is composed of a dipole magnet instrumented with resistive plate chambers and drift tubes. Each target section has 31 brick walls interleaved with orthogonal pairs of scintillator strip planes that compose the Target Tracker (TT). A detailed description of the OPERA detector can be found in [16].

A brick consists of 57 emulsion films interleaved with 56 lead plates of $1 \mathrm{~mm}$ thickness. The films are made of 2 emulsion layers, each $44 \mu \mathrm{m}$ thick coated on both sides of a $205 \mu \mathrm{m}$ transparent plastic base. The brick has transverse dimensions of $128 \times 103 \mathrm{~mm}^{2}$, a thickness of $81 \mathrm{~mm}$ (about 10 radiation lengths) and it weighs $8.3 \mathrm{~kg}$. A pair of removable emulsion films called changeable sheets (CS) attached to the downstream face of each brick act as interfaces between the emulsion films in the brick and the TT. There are about 150,000 bricks in total for a target mass of 1.25 ktons.

TT hit patterns are used to identify the bricks possibly containing the neutrino interaction vertex [17]. The most probable brick is then extracted from the target and its CS films scanned. If a signal compatible with the TT predictions is found, the brick is disassembled and its films analysed. Once the vertex has been located, a surrounding volume of about $2 \mathrm{~cm}^{3}$ is scanned to determine the event topology. Otherwise, the procedure is repeated in the next brick in the probability 


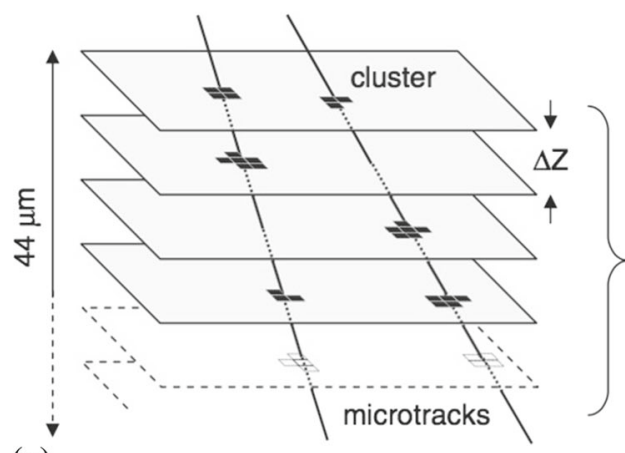

(a)

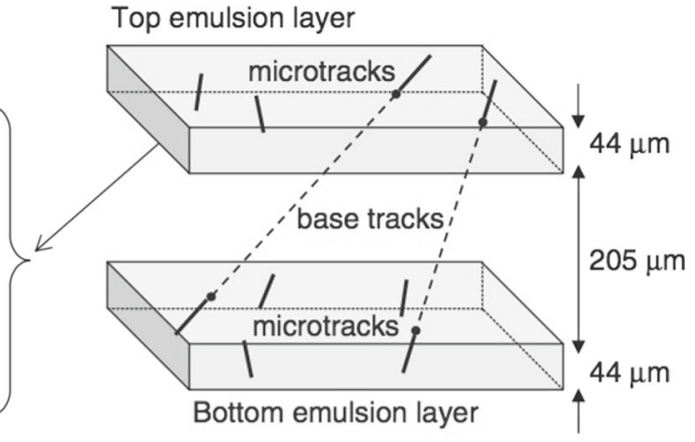

(b)

Fig. 1 a Combination of clusters belonging to images taken at different depths in one emulsion layer forms a micro-track; $\mathbf{b}$ the association of two micro-tracks across the plastic base forms a base-track

ranking. Track recognition in an emulsion layer is based on 16 tomographic images taken by the sensor of an automated microscope and equally spaced through the $44-\mu \mathrm{m}$ depth of the layer. A sequence of aligned grains in a layer forms a micro-track (Fig. 1a) and the association of two matching micro-tracks on each side of the plastic base in a film constitutes a base-track (Fig. 1b). Track positions and slopes are determined by a linear fit through base-tracks in the analysed volume. The details of the event analysis procedure are described in [18].

\section{Analysis}

During the physics runs between 2008 and 2012, OPERA collected data corresponding to $1.8 \times 10^{20}$ protons on target. The electronic detectors recorded 19,505 neutrino interactions in the target fiducial volume. The search of the neutrino vertex in the first and second most probable bricks plus some additional selections (see [18] for more details) resulted in a sample of 5603 located events out of which 4406 have an identified muon. For the present measurement an unbiased sub-sample of 818 events occurring in the lead with a negatively charged muon identified by the muon spectrometer was selected in order to measure the track and vertex parameters in the target including a detailed check of the nuclear break-up and evaporation processes.

It is further required that $W^{2}$, the square of the invariant mass of the hadronic system measured with the electronic detector, is larger than $1 \mathrm{GeV}^{2} / \mathrm{c}^{4}$ in order to eliminate the quasi-elastic contribution. The final data sample contains 795 events with an identified muon. The contamination from $v_{\mu}$ neutral-current interactions in the final data sample is estimated by MC simulations to be less than $1 \%$.

Selected $v_{\mu}$ CC events are inspected carefully and tracks are classified as being left by a minimum ionisation particle (mip), grey and black depending on their ionisation features. The mip tracks are left by the muon and by highly relativistic

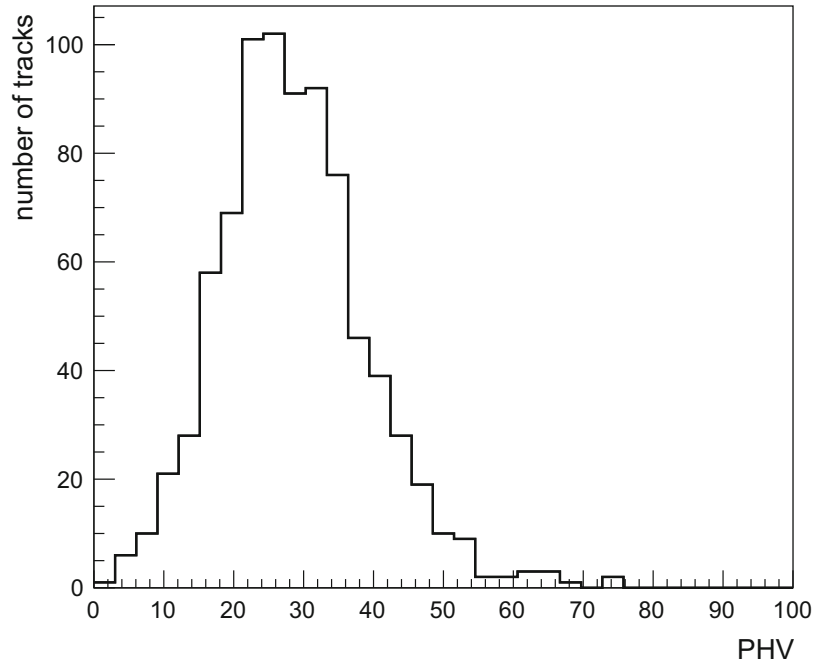

Fig. 2 Pulse height volume distribution of muon tracks

charged hadrons resulting from the cascade of interactions generated inside the target nucleus by the primary hadrons emitted with the muon at the neutrino-nucleon interaction. Black tracks are produced by low energy fragments (protons, deuterons, alpha particles and heavier fragments) emitted from the excited target nucleus. Black tracks are classified as backward or forward based on the emission direction. Grey tracks are left by slow particles which are interpreted as being recoil nucleons emitted during the nuclear cascade [19].

The black tracks are easy to recognise visually since they are heavily ionising, they have short path lengths and stop within two lead plates. The separation between mip and grey tracks is based on the pulse height volume (PHV) [20,21] which is defined as the sum of the number of pixels associated with each track in all sixteen layers of images [22,23]. PHV indicates the track width and is therefore a measure of the grain density of a track that reflects the energy deposition of a particle in the emulsion film. The PHV distribution of muon tracks is shown in Fig. 2. All muon tracks have a PHV below 


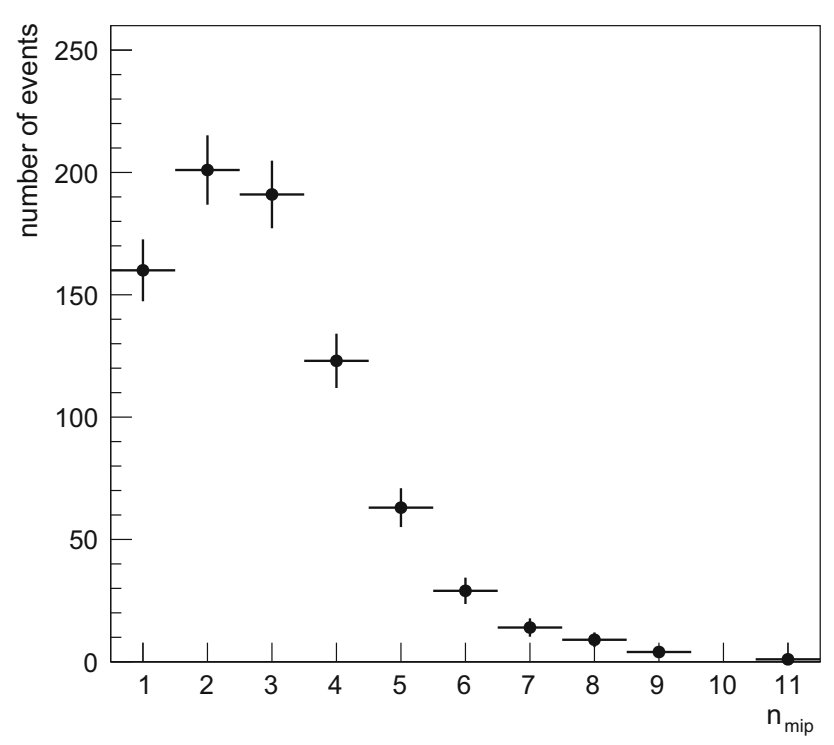

Fig. 3 Multiplicity distribution of mip tracks

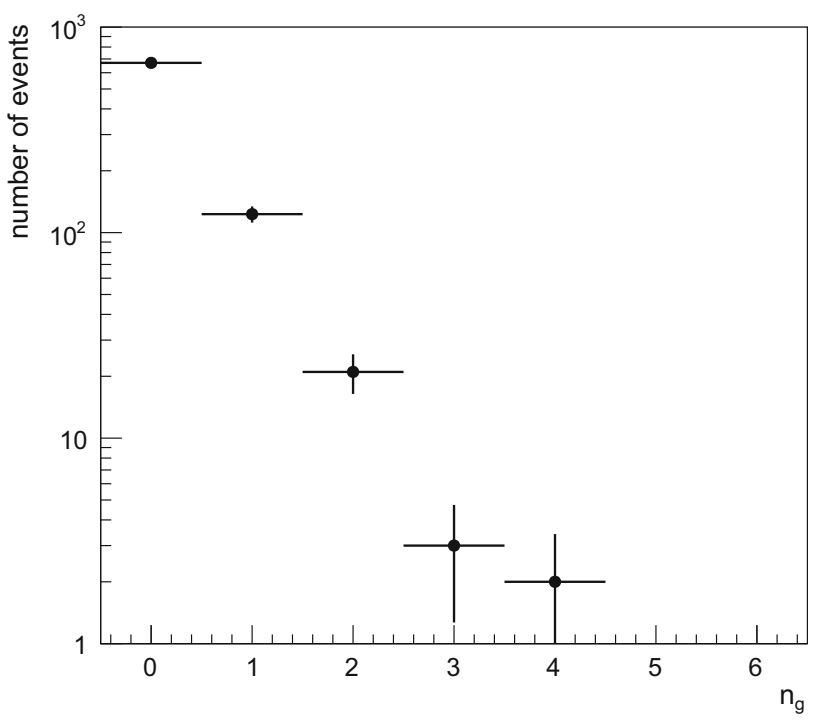

Fig. 4 Multiplicity distribution of grey tracks

85 and mip tracks are defined by requiring a PHV smaller than 85 , the tracks with a higher PHV being classified as grey. The mip, grey and black track multiplicities are shown in Figs. 3, 4 and 5, respectively. The average numbers of mip and grey tracks in $v_{\mu} C C$ events are $\left\langle n_{\text {mip }}\right\rangle=2.94 \pm 0.05$ and $\left\langle n_{g}\right\rangle=0.22 \pm 0.01$, respectively. The average number of backward and forward black tracks are measured to be $\left\langle n_{b}\right\rangle_{B}=0.15 \pm 0.01$ and $\left\langle n_{b}\right\rangle_{F}=0.38 \pm 0.02$, respectively. The charged hadrons multiplicity $n_{c h}$ is defined as the number of mip tracks excluding the muon track, its average being $\left\langle n_{c h}\right\rangle=\left\langle n_{\text {mip }}\right\rangle-1=1.94 \pm 0.05$. The distribution of mip tracks as a function of their emission angle with respect to the beam axis is given separately for muons and charged hadrons in Table 1.

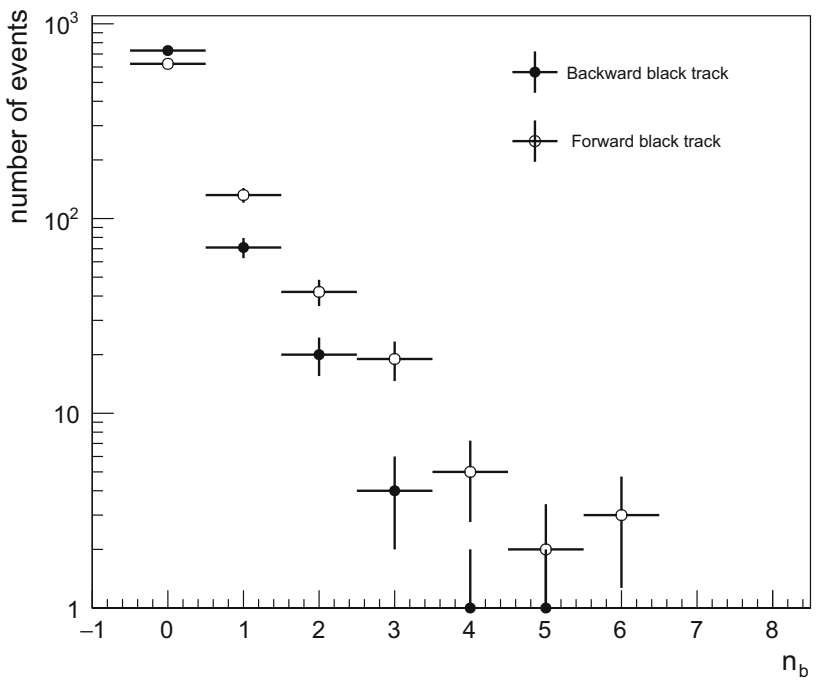

Fig. 5 Multiplicity distribution of backward and forward black tracks

Table 1 The numbers of hadron $\left(n_{c h}\right)$ and muon $\left(n_{\mu}\right)$ tracks per event as a function of the emission angle $\theta$

\begin{tabular}{llll}
\hline$\theta$ (Radian) & $\langle\theta\rangle$ & $n_{c h} /$ event & $n_{\mu}$ /event \\
\hline $0.00 \div 0.050$ & $0.031 \pm 0.001$ & 0.134 & 0.070 \\
$0.050 \div 0.100$ & $0.076 \pm 0.001$ & 0.258 & 0.195 \\
$0.100 \div 0.150$ & $0.126 \pm 0.001$ & 0.280 & 0.231 \\
$0.150 \div 0.200$ & $0.174 \pm 0.001$ & 0.237 & 0.177 \\
$0.200 \div 0.300$ & $0.246 \pm 0.002$ & 0.383 & 0.195 \\
$0.300 \div 0.400$ & $0.347 \pm 0.003$ & 0.298 & 0.079 \\
$0.400 \div 0.500$ & $0.450 \pm 0.004$ & 0.179 & 0.029 \\
$0.500 \div 0.600$ & $0.549 \pm 0.006$ & 0.113 & 0.017 \\
$\geq 0.600$ & $0.661 \pm 0.03$ & 0.058 & 0.002 \\
Total & & 1.94 & 1.00 \\
\hline
\end{tabular}

\subsection{Efficiency estimation}

The location and reconstruction efficiency is computed using the standard OPERA simulation framework. The neutrino fluxes and spectra are based on the FLUKA simulation [24] of the CNGS beam-line. The neutrino interactions in the detector are generated using the NEGN generator [26]. MC-generated $v_{\mu} C C$ events are processed through the full OPERA simulation chain, from the event classification and brick finding provided by the electronics detectors to the CS analysis and event location and analysis in the brick.

The location and reconstruction efficiency, shown in Table 2, of the $v_{\mu} C C$ events is estimated as a function of $W^{2}$ and of the charged hadron multiplicity. Since the event location is done using mip tracks, the location efficiency does not depend on the black and grey track multiplicities at the neutrino interaction vertex. Figure 6 shows the good agreement between the charged hadrons multiplicities obtained for 
Table 2 Location and reconstruction efficiency as a function of $\mathrm{W}^{2}$ and the charged hadron $n_{c h}$ multiplicity

\begin{tabular}{|c|c|c|c|c|c|c|c|}
\hline \multirow[t]{2}{*}{$W^{2}\left(\mathrm{GeV}^{2} / \mathrm{c}^{4}\right)$} & \multicolumn{7}{|l|}{$n_{c h}$} \\
\hline & 0 & 1 & 2 & 3 & 4 & $\geq 5$ & Total \\
\hline $1-3$ & $0.32 \pm 0.03$ & $0.48 \pm 0.02$ & $0.53 \pm 0.04$ & $0.55 \pm 0.06$ & $0.56 \pm 0.09$ & $0.62 \pm 0.17$ & $0.46 \pm 0.01$ \\
\hline $3-6$ & $0.34 \pm 0.02$ & $0.47 \pm 0.02$ & $0.54 \pm 0.03$ & $0.56 \pm 0.04$ & $0.65 \pm 0.07$ & $0.66 \pm 0.11$ & $0.47 \pm 0.01$ \\
\hline $6-9$ & $0.33 \pm 0.02$ & $0.46 \pm 0.02$ & $0.56 \pm 0.02$ & $0.63 \pm 0.03$ & $0.67 \pm 0.06$ & $0.68 \pm 0.08$ & $0.48 \pm 0.01$ \\
\hline $9-12$ & $0.34 \pm 0.02$ & $0.49 \pm 0.02$ & $0.57 \pm 0.02$ & $0.62 \pm 0.03$ & $0.69 \pm 0.05$ & $0.66 \pm 0.07$ & $0.50 \pm 0.01$ \\
\hline $12-15$ & $0.36 \pm 0.02$ & $0.50 \pm 0.02$ & $0.58 \pm 0.02$ & $0.63 \pm 0.03$ & $0.70 \pm 0.04$ & $0.70 \pm 0.06$ & $0.52 \pm 0.01$ \\
\hline $15-19$ & $0.37 \pm 0.02$ & $0.49 \pm 0.02$ & $0.60 \pm 0.02$ & $0.62 \pm 0.02$ & $0.66 \pm 0.03$ & $0.74 \pm 0.05$ & $0.53 \pm 0.01$ \\
\hline $19-25$ & $0.40 \pm 0.02$ & $0.52 \pm 0.02$ & $0.56 \pm 0.02$ & $0.64 \pm 0.02$ & $0.66 \pm 0.03$ & $0.68 \pm 0.04$ & $0.54 \pm 0.01$ \\
\hline $25-35$ & $0.41 \pm 0.02$ & $0.56 \pm 0.02$ & $0.56 \pm 0.02$ & $0.60 \pm 0.02$ & $0.62 \pm 0.02$ & $0.69 \pm 0.03$ & $0.55 \pm 0.01$ \\
\hline$\geq 35$ & $0.39 \pm 0.02$ & $0.39 \pm 0.02$ & $0.42 \pm 0.01$ & $0.43 \pm 0.01$ & $0.54 \pm 0.02$ & $0.57 \pm 0.02$ & $0.45 \pm 0.01$ \\
\hline Total & $0.37 \pm 0.007$ & $0.48 \pm 0.007$ & $0.54 \pm 0.008$ & $0.57 \pm 0.09$ & $0.62 \pm 0.01$ & $0.62 \pm 0.01$ & $0.50 \pm 0.01$ \\
\hline
\end{tabular}



Fig. 6 Comparison between the PDF of the data and MC charged hadron multiplicities

observed and MC simulated data. The efficiency correction is hereafter applied to the measured data distributions.

In $v_{\mu} C C$ interactions, neglecting the Fermi motion, the full kinematics of the event can be reconstructed from the measurement of the muon momentum $p_{\mu}$, its angle $\theta_{\mu}$ with respect to the beam axis, and $E_{\text {had }}$, the energy transfer to the hadronic system, obtained by measuring the energy deposition in the TT (detailed information is available in [16])

$$
\begin{aligned}
E_{v} & =E_{\mu}+E_{\text {had }} \\
Q_{v}^{2} & =2 E_{v}\left(E_{\mu}-p_{\mu} \cos \theta_{\mu}\right)-m_{\mu}^{2} \\
W^{2} & =2 m_{N} E_{\text {had }}+m_{N}^{2}-Q_{v}^{2},
\end{aligned}
$$

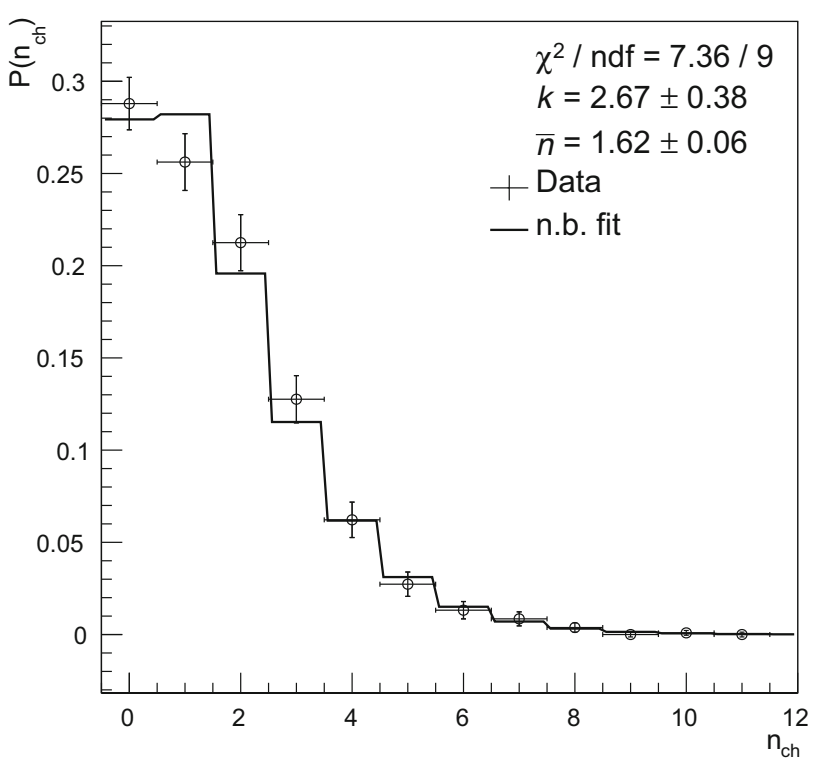

Fig. 7 The multiplicity distribution corrected for efficiencies superimposed with the negative binomial distribution (n.b.) defined in Eq. 1

where $E_{v}$ and $E_{\mu}$ are the energy of the incoming neutrino and the muon, $Q_{v}^{2}$ the squared four-momentum transfer, $m_{N}$ and $m_{\mu}$ are the mass of the nucleon and muon respectively. The average neutrino energy of the data sample is $\left\langle E_{v}\right\rangle=$ $(19.6 \pm 0.3) \mathrm{GeV}$, the mean-square momentum transferred to the hadronic system is $\left\langle Q_{v}^{2}\right\rangle=(5.7 \pm 0.3) \mathrm{GeV}^{2} / \mathrm{c}^{2}$, and the mean-square of the invariant mass of the hadronic system is $\left\langle W^{2}\right\rangle=(16.9 \pm 0.6) \mathrm{GeV}^{2} / \mathrm{c}^{4}$.

\subsection{Multiplicity distributions}

Figure 7 displays the multiplicity distribution after correction for efficiencies. It is observed that it is well described by a negative binomial pdf, also shown on the figure, 


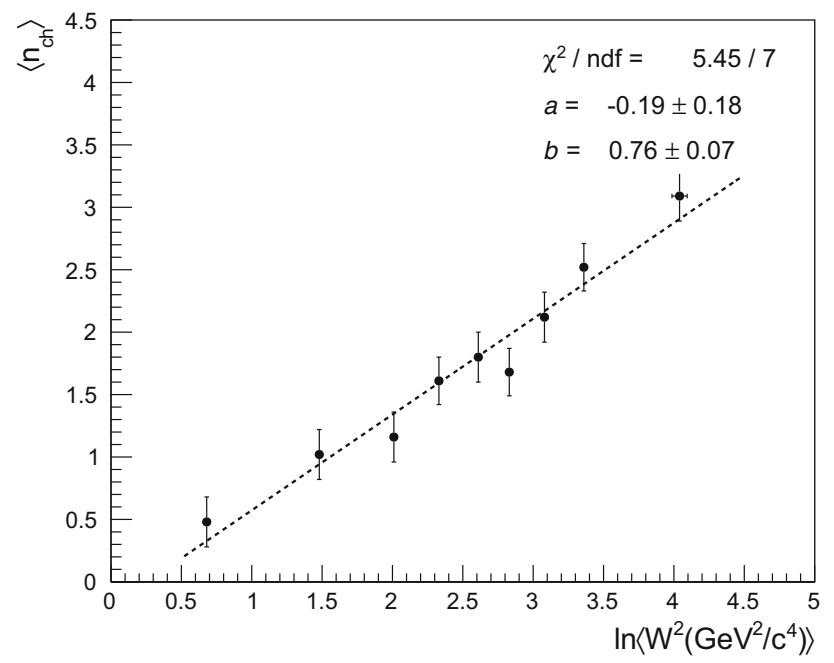

Fig. 8 The average charged hadron multiplicity distributions as a function of $\ln W^{2}$

$P\left(n_{c h}\right)=\left(\begin{array}{c}n_{c h}+k-1 \\ n_{c h}\end{array}\right) p^{n_{c h}}(1-p)^{k}$

with $p=\frac{\bar{n} / k}{1+\bar{n} / k}$, the mean $\bar{n}=1.62 \pm 0.06$ fully compatible with its measured value $<n_{c h}>=1.63$ and parameter $k=$ $2.67 \pm 0.38$. The $\chi^{2} / n d f$ of the fit is $7.36 / 9$.

The average charged hadrons multiplicity as a function of $W^{2}$ is presented in Fig. 8. The data is well described by a linear function in $\ln W^{2}$ : $\left\langle n_{c h}\right\rangle=a+b \ln W^{2}$.

The values of the fitted parameters are $a=-0.19 \pm 0.18$ and $b=0.76 \pm 0.07$. Values for different $W^{2}$ bin intervals are shown in Table 3. A comparison with other experiments is given in Table 4.

\subsection{Dispersion}

One of the characteristics of the multiplicity distribution which is of considerable theoretical interest is its dispersion. In this section we investigate its dependence on the average multiplicity. The dispersion $D_{c h}$ is defined as $D_{c h}=$ $\sqrt{\left\langle n_{c h}^{2}\right\rangle-\left\langle n_{c h}\right\rangle^{2}}$. For independent particle production it would follow a Poisson distribution with $D_{c h}=\sqrt{\left\langle n_{c h}\right\rangle}$. However, it was observed that charged particles production in hadronic interactions satisfies an empirical parameterisation [27]:

$D_{c h}=A+B\left\langle n_{c h}\right\rangle$

Figure 9 shows the dependence of the dispersion on the average multiplicity $\left\langle n_{c h}\right\rangle$ with a linear fit superimposed. The values of the fitted parameters are $A=0.59 \pm 0.12$ and $B=0.46 \pm 0.06$. They are shown in Table 5 together with those obtained in other experiments for comparison.

Table 3 The charged hadron multiplicity $n_{c h}$ distribution as a function of $W^{2}$ (efficiency corrections are not applied and errors shown are statistical only)

\begin{tabular}{|c|c|c|c|c|c|c|c|c|c|c|c|c|c|c|c|}
\hline \multirow[t]{2}{*}{$W^{2}\left(\mathrm{GeV}^{2} / \mathrm{c}^{4}\right)$} & \multirow[t]{2}{*}{$\ln \left\langle W^{2}\left(\mathrm{GeV}^{2} / \mathrm{c}^{4}\right)\right\rangle$} & \multicolumn{14}{|l|}{$n_{c h}$} \\
\hline & & 0 & 1 & 2 & 3 & 4 & 5 & 6 & 7 & 8 & 9 & 10 & $\geq 11$ & $\left\langle n_{c h}\right\rangle$ & Total \\
\hline $1-3$ & $0.68 \pm 0.03$ & 59 & 21 & 7 & 1 & 0 & 1 & 0 & 0 & 0 & 0 & 0 & 0 & $0.48 \pm 0.11$ & 89 \\
\hline $3-6$ & $1.48 \pm 0.01$ & 29 & 37 & 24 & 4 & 2 & 0 & 1 & 0 & 0 & 0 & 0 & 0 & $1.14 \pm 0.10$ & 97 \\
\hline $6-9$ & $2.01 \pm 0.01$ & 28 & 37 & 16 & 12 & 5 & 1 & 1 & 0 & 0 & 0 & 0 & 0 & $1.36 \pm 0.12$ & 100 \\
\hline $9-12$ & $2.33 \pm 0.01$ & 10 & 26 & 25 & 13 & 6 & 2 & 1 & 0 & 0 & 0 & 0 & 0 & $1.86 \pm 0.13$ & 83 \\
\hline $12-15$ & $2.61 \pm 0.01$ & 10 & 24 & 29 & 27 & 3 & 2 & 3 & 0 & 0 & 0 & 0 & 0 & $2.07 \pm 0.13$ & 98 \\
\hline $15-19$ & $2.83 \pm 0.01$ & 12 & 17 & 35 & 14 & 8 & 4 & 1 & 1 & 0 & 0 & 0 & 0 & $2.10 \pm 0.15$ & 92 \\
\hline $19-25$ & $3.08 \pm 0.01$ & 5 & 18 & 22 & 19 & 12 & 3 & 1 & 3 & 0 & 0 & 0 & 0 & $2.51 \pm 0.16$ & 83 \\
\hline $25-35$ & $3.36 \pm 0.01$ & 4 & 15 & 17 & 19 & 12 & 8 & 2 & 1 & 1 & 0 & 0 & 0 & $2.79 \pm 0.18$ & 79 \\
\hline$\geq 35$ & $4.04 \pm 0.05$ & 3 & 6 & 16 & 14 & 15 & 8 & 4 & 4 & 3 & 0 & 1 & 0 & $3.59 \pm 0.24$ & 74 \\
\hline Total & $2.83 \pm 0.03$ & 160 & 201 & 191 & 123 & 63 & 29 & 14 & 9 & 4 & 0 & 1 & 0 & $1.94 \pm 0.05$ & 795 \\
\hline
\end{tabular}

Table 4 Values of the parameters of the linear fit to the average charged hadrons multiplicity dependence on $\ln W^{2}$. The results from other experiments are also shown for comparison

\begin{tabular}{lllll}
\hline Reaction & $\left\langle E_{v}\right\rangle(\mathrm{GeV})$ & $\mathrm{a}$ & $\mathrm{b}$ & Ref. \\
\hline$v_{\mu}$-emulsion & 38 & $0.45 \pm 0.24$ & $0.94 \pm 0.08$ & {$[7]$} \\
$v_{\mu}$-emulsion & 50 & $1.92 \pm 0.68$ & $1.19 \pm 0.23$ & {$[8]$} \\
$v_{\mu}$-emulsion & 8.7 & $1.07 \pm 0.05$ & $1.32 \pm 0.11$ & {$[9,10]$} \\
$v_{\mu}$-lead & 20 & $-0.19 \pm 0.18$ & $0.76 \pm 0.07$ & OPERA
\end{tabular}




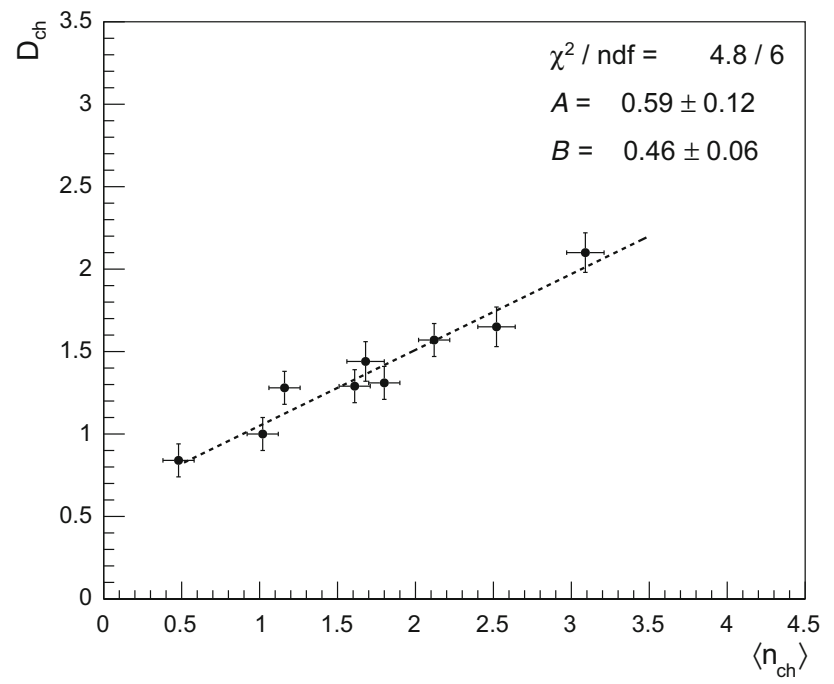

Fig. 9 The charged hadrons multiplicity dispersion as a function of $\left\langle n_{c h}\right\rangle$

Table 5 Values of parameters $A$ and $B$ obtained by a linear fit on the distribution of $D_{c h}$ versus $\left\langle n_{c h}\right\rangle$. The results obtained in other neutrino experiments are also shown

\begin{tabular}{lllll}
\hline Reaction & $\left\langle E_{v}\right\rangle(\mathrm{GeV})$ & $A$ & $B$ & Ref. \\
\hline$v_{\mu}$-emulsion & 38 & $1.18 \pm 0.17$ & $0.20 \pm 0.05$ & {$[7]$} \\
$v_{\mu}$-p & $>5$ & $0.36 \pm 0.03$ & $0.36 \pm 0.03$ & {$[6]$} \\
$v_{\mu}$-lead & 20 & $0.59 \pm 0.12$ & $0.46 \pm 0.06$ & OPERA \\
\hline
\end{tabular}

\subsection{KNO scaling}

Koba, Nielsen, and Olesen have shown that the multiplicity distribution $P\left(n_{c h}\right)$ scaled by the average multiplicity $\left\langle n_{c h}\right\rangle$ is asymptotically independent of the primary energy and depends only on variable $z=\frac{n_{c h}}{\left\langle n_{c h}\right\rangle}$ :

$\left\langle n_{c h}\right\rangle . P\left(n_{c h}\right) \stackrel{E \rightarrow \infty}{\longrightarrow} \Psi(z)$

KNO scaling is derived from Feynman scaling, i.e. based on the assumption that the rapidity density $\frac{d n_{c h}}{d y}$ reaches its limit value at $y=0$ above a certain energy which corresponds to an asymptotic scaling of the total multiplicity as $\left\langle n_{c h}\right\rangle \propto$ $\ln \sqrt{s}$. KNO scaling implies that the intercept $A$ in Eq. 3 be compatible with 0 , which is not the case at low to medium energies for all kinds of interactions. Buras et al. [28] have introduced a new variable $z^{\prime}$ defined as

$z^{\prime}=\frac{n_{c h}-\alpha}{\left\langle n_{c h}-\alpha\right\rangle}$,

where the reaction dependent and energy independent parameter $\alpha$ is chosen in order to provide an extension of the KNO



Fig. $10 \mathrm{KNO}$ scaling distribution. The curve superposed on the data results from a fit using the parameterisation defined in Eq. 7

scaling to low energies. In the current analysis, this implies that $\alpha=-A / B=-1.28$. Hence

$\left\langle\left(n_{c h}-\alpha\right)\right\rangle P\left(n_{c h}\right)=\Psi\left(z^{\prime}\right)$.

A tentative explanation for a non-zero value for $\alpha$ has been proposed in terms of a leading particle effect in interactions of hadrons [28] and of neutrinos [29] as well as resulting from the heavy nuclear targets in neutrinos experiments using emulsion [7]. Figure 10 shows the distributions obtained for $\Psi$ as a function of $z^{\prime}$ for three different intervals of $W^{2}$ for $v_{\mu}$ lead CC interactions. The data shows good agreement with approximate KNO scaling. Such observation has been made by other experiments [7,29-32]. The fitted curve superimposed on the data results from a parameterisation of $\Psi\left(z^{\prime}\right)$ of the type first introduced by Slattery [33]:

$\Psi\left(z^{\prime}\right)=\left(A\left(z^{\prime}\right)^{3}+B\left(z^{\prime}\right)^{4}\right) e^{-C z^{\prime}}$

The values obtained for the fit parameters are $\mathrm{A}=32.16 \pm$ 5.35, $\mathrm{B}=-10.18 \pm 1.68$ and $\mathrm{C}=3.31 \pm 0.11$. The $\chi^{2} / n d f$ of the fit and the correlation matrix of the parameters are $14.7 / 23$ and

$$
\left(\begin{array}{ccc}
1.000 & -0.962 & 0.866 \\
-0.962 & 1.000 & -0.744 \\
0.866 & -0.744 & 1.000
\end{array}\right),
$$

respectively.

\section{Conclusion}

In this article, we studied the characteristics of the multiplicity distribution of charged hadrons in neutrino-lead 
interactions in the OPERA detector with the main objective to aid in tuning the models used in MC event generators. For this purpose, the results are presented in detail in the form of tables. They can be summarized as follow:

i The dependence of the average multiplicity $\left\langle n_{c h}\right\rangle$ on $\ln W^{2}$ is approximately linear.

ii The dependence on the charged hadrons multiplicity $n_{c h}$ of its dispersion $D_{c h}$ is approximately linear.

iii Approximate $\mathrm{KNO}$ scaling is valid for the charged hadrons multiplicity.

Acknowledgements We acknowledge CERN for the successful operation of the CNGS facility and INFN for the continuous support given to the experiment through its LNGS laboratory. We acknowledge funding from our national agencies: Fonds de la Recherche ScientifiqueFNRS and Institut Interuniversitaire des Sciences Nucleaires for Belgium; MoSES for Croatia; CNRS and IN2P3 for France; BMBF for Germany; INFN for Italy; JSPS, MEXT, the QFPU-Global COE program of Nagoya University, and Promotion and Mutual Aid Corporation for Private Schools of Japan for Japan; SNF, the University of Bern and ETH Zurich for Switzerland; the Russian Foundation for Basic Research (Grant no. 12-02-12142 ofim), the Programs of the Presidium of the Russian Academy of Sciences (Neutrino Physics and Experimental and Theoretical Researches of Fundamental Interactions), and the Ministry of Education and Science of the Russian Federation for Russia, the Basic Science Research Program through the National Research Foundation of Korea (NRF) funded by the Ministry of Science, ICT and Future Planning (Grant no. NRF-2015R1A2A 2A01004532) for Korea; and TUBITAK, the Scientific and Technological Research Council of Turkey for Turkey (Grant no. 108T324). We thank the IN2P3 Computing Centre (CC-IN2P3) for providing computing resources.

Open Access This article is distributed under the terms of the Creative Commons Attribution 4.0 International License (http://creativecomm ons.org/licenses/by/4.0/), which permits unrestricted use, distribution, and reproduction in any medium, provided you give appropriate credit to the original author(s) and the source, provide a link to the Creative Commons license, and indicate if changes were made. Funded by SCOAP ${ }^{3}$.

\section{References}

1. K.J. Biebl et al., Fortschr. Phys. V28, 124 (1980)

2. G.J. Alner et al., Phys. Rep. 154, 247 (1987)

3. R.E. Ansorge et al., Z. Phys. C43, 357 (1989)

4. C. Berger et al., (PLUTO Coll.) Phys. Lett. B78, 176 (1978)

5. W. Brandelik et al., (TASSO Coll.) Phys. Lett. B89, 418 (1980)

6. P. Allen et al., Nucl. Phys. B181, 385 (1981)

7. A. Kayis-Topaksu et al., (CHORUS Coll.), Eur. Phys. J. C51, 775 (2007)

8. L. Voyvodik et al., ITEP-86-91, Moscow (1986)

9. YuD Aleshin et al., Zh. Eksp. Teor. Fiz. 110, 391 (1996)

10. YuD Aleshin et al., JTEP 83, 208 (1996)

11. F. Koba, H.B. Nielsen, P. Olesen, Nucl. Phys. B40, 317 (1972)

12. K. Elsener, Reports No. CERN 98-02 and No. INFN/AE-98/05 (1998)

13. R. Bailey et al., Reports No. CERN-SL-99-034-DI and No. INFNAE-99- 05, 1999, addendum to Reports No. CERN 98-02 and No. INFN-AE-98-05 (1998)

14. CNGS Web site. http://proj-cngs.web.cern.ch/proj-cngs

15. N. Agafonova et al. (OPERA Coll.), PRL 115, 121802 (2015)

16. R. Acquafredda et al., (OPERA Coll.), JINST 4, P04018 (2009)

17. YuA Gornushkin, S.G. Dmitrievsky, A.V. Chukanov, Phys. Part. Nucl. Lett. 12, 89 (2015)

18. N. Agafonova et al., (OPERA Coll.), J. High Energy Phys. 11, 036 (2013)

19. C.F. Powell, P.H. Fowler, D.H. Perkins, The study of elementary particles by the photographic method (Pergamon Press, London, 1959)

20. T. Toshito et al., Nucl. Instrum. Meth. A556, 482 (2006)

21. J. Shin et al., Nucl. Instrum. Meth. B346, 201 (2015)

22. K. Morishima, T. Nakano, JINST 5, P04011 (2010)

23. C. Bozza et al., Nucl. Instrum. Meth. A 703, 204 (2013)

24. T.T. Bohlen et al., Nucl. Data Sheets 120, 211-214 (2014)

25. A. Ferrari, P.R. Sala, A. Fasso, J. Ranft, CERN-2005-10, INFNTC-05-11, SLAC-R-773 (2005)

26. D. Autiero, Nucl. Phys. B 139(Proc. Suppl.), 253 (2005)

27. R.K. Shivpuri, V.K. Verma, Z. Phys. C58, 7 (1993)

28. A.J. Buras et al., Phys. Lett. B47, 251 (1973)

29. D.S. Baranov et al., Z. Phys. C21, 189 (1984)

30. J. Hebert et al., Phys. Rev. D15, 1867 (1977)

31. J. Hebert et al., Phys. Lett. B48, 467 (1974)

32. A. Wroblewski, Acta Phys. Polon B4, 857 (1973)

33. P. Slattery, Phys. Rev. D7, 2073 (1973) 